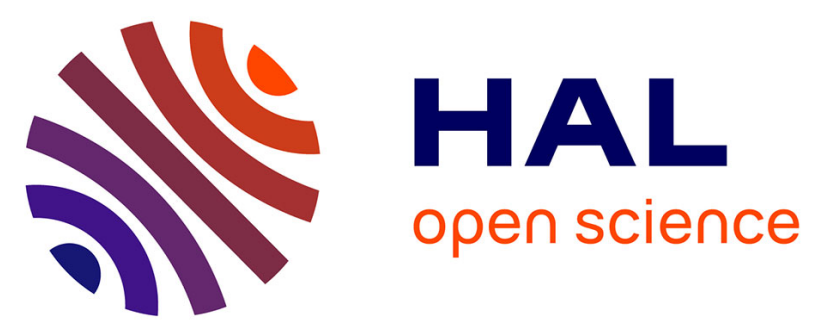

\title{
Apomorphine en perfusion sous-cutanée continue dans la maladie de Parkinson : le point de vue pharmacologique
}

\author{
Manon Auffret, Sophie Drapier, Marc Verin, Paul Sauleau
}

\section{To cite this version:}

Manon Auffret, Sophie Drapier, Marc Verin, Paul Sauleau. Apomorphine en perfusion sous-cutanée continue dans la maladie de Parkinson : le point de vue pharmacologique. Pratique Neurologique FMC, 2016, 7 (1), pp.25-32. 10.1016/j.praneu.2016.01.006 . hal-01295662

HAL Id: hal-01295662

https://hal-univ-rennes1.archives-ouvertes.fr/hal-01295662

Submitted on 22 Jul 2016

HAL is a multi-disciplinary open access archive for the deposit and dissemination of scientific research documents, whether they are published or not. The documents may come from teaching and research institutions in France or abroad, or from public or private research centers.
L'archive ouverte pluridisciplinaire HAL, est destinée au dépôt et à la diffusion de documents scientifiques de niveau recherche, publiés ou non, émanant des établissements d'enseignement et de recherche français ou étrangers, des laboratoires publics ou privés. 


\section{Apomorphine en perfusion sous-cutanée continue dans la maladie de Parkinson : le point de vue pharmacologique}

Manon Auffret (PharmD, MSc) $)^{1}$, Sophie Drapier (MD) ${ }^{1,2}$, Marc Vérin (MD, PhD $)^{1,2}$, Paul Sauleau (MD, PhD) $)^{1,3}$

1 : EA 4712 "Comportement et Noyaux Gris Centraux" - Université de Rennes 1

2 : Service de Neurologie - CHU de Rennes

3 : Service de Neurophysiologie - CHU de Rennes

\section{Résumé}

Agoniste dopaminergique le plus ancien et le plus puissant, l'apomorphine n'en reste pas moins sous-utilisée dans la maladie de Parkinson, malgré une efficacité reconnue. Cet article fournit des éléments de compréhension de la pharmacologie et de la pharmacocinétique de cette molécule, à même d'expliquer les effets cliniques ainsi que les différences d'efficacité entre les individus. Pour le clinicien, ces éléments sont d'une importance majeure pour comprendre les indications de l'apomorphine et sa place dans la pharmacopée antiparkinsonienne, dans l'objectif de personnaliser et d'optimiser le traitement pour chaque patient.

\section{Abstract}

Subcutaneous infusion with apomorphine is one of the treatments available for Parkinson's disease. Even if increasing evidence of its efficacy on both motor and non-motor symptoms is found in literature, it is still underused. This article reviews pharmacological and pharmacokinetic properties of apomorphine and its impact on clinical decision. Despite their complexity, those properties are a key to understand the effect of the drug and its variability of efficacy and dosage amongst patients.

\section{Mots clés}

Apomorphine - Pharmacologie - Récepteurs dopaminergiques - Maladie de Parkinson

\section{Key words}

Apomorphine - Pharmacology - Dopaminergic receptors - Parkinson's disease

\section{Introduction}

Considérée à ce jour comme l'agoniste dopaminergique le plus ancien et le plus puissant (Antonini, 2009 ; Chaudhuri et al, 2013), l'apomorphine est un dérivé de la molécule de morphine (Matthiessen et Wright, 1869). Contrairement à celle-ci, l'apomorphine n'est pas un alcalö̈de présent naturellement dans le Papaver somniferum ; elle fut synthétisée pour la première fois en 1869 par l'action conjuguée 
de la chaleur $\left(100^{\circ} \mathrm{C}\right)$ et d'une solution acide (e.g : acide sulfurique, acide chlorhydrique $\mathrm{HCl}$ ou $\mathrm{ZnCl}_{2}$ ) sur la molécule de morphine. Cette réaction chimique induisant une transformation spatiale qui rend théoriquement impossible une interaction entre l'apomorphine et les récepteurs morphiniques, il est classiquement admis que l'apomorphine ne possède pas les propriétés narcotiques et stupéfiantes de la morphine, bien qu'elle soit sédative (LeWitt, 2004 ; Chen et al, 2005 ; Drapier et Vérin, 2006 ; Zagnoli et Rouhart, 2006 ; Haq et al, 2007). Historiquement, l'apomorphine reste le premier agoniste dopaminergique utilisé dans la maladie de Parkinson (MP) (Schwab et al, 1951).

Aujourd'hui, elle reste sous-utilisée malgré une efficacité reconnue. Cet article vise à apporter des éléments de compréhension de la pharmacocinétique et du métabolisme de l'apomorphine, qui, bien que de prime abord complexes, sont à même d'expliquer les différences notables d'efficacité et de tolérance entre individus (Van der Geest et al, 1997 ; Garrido et al, 2002 ; LeWitt, 2004). Ces éléments peuvent paraître quelque peu éloignés de la pratique au lit du malade, mais ils sont essentiels pour anticiper les effets biologiques et cliniques de l'apomorphine, qu'ils soient bénéfiques ou indésirables, et ainsi optimiser le traitement de chaque patient.

\section{Structure et propriétés de l'apomorphine}

Appartenant à la classe des (di)benzoquinolines (Menon et Stacy, 2007 ; Ribaric, 2012), solide gris-blanc, soluble dans l'éthanol et l'acétone, l'eau et le benzène, insoluble dans le chloroforme (Argiolas et Hedlund, 2001), l'apomorphine est soluble dans les solutions salines (Deleu et al, 2004 ; Factor, 2004) et de fait adaptée à une administration sous forme injectable.

Le chlorhydrate d'apomorphine (6-methyl-6aß-noraporphine-10,11-diol; $\mathrm{C}_{17} \mathrm{H}_{17} \mathrm{NO}_{2}$ ) est donc un dérivé de la molécule de morphine, polycyclique, au poids moléculaire de 267.32 daltons (Haq et al, 2007), obtenu par l'action conjuguée de la chaleur et d'une solution acide (Figure 1).

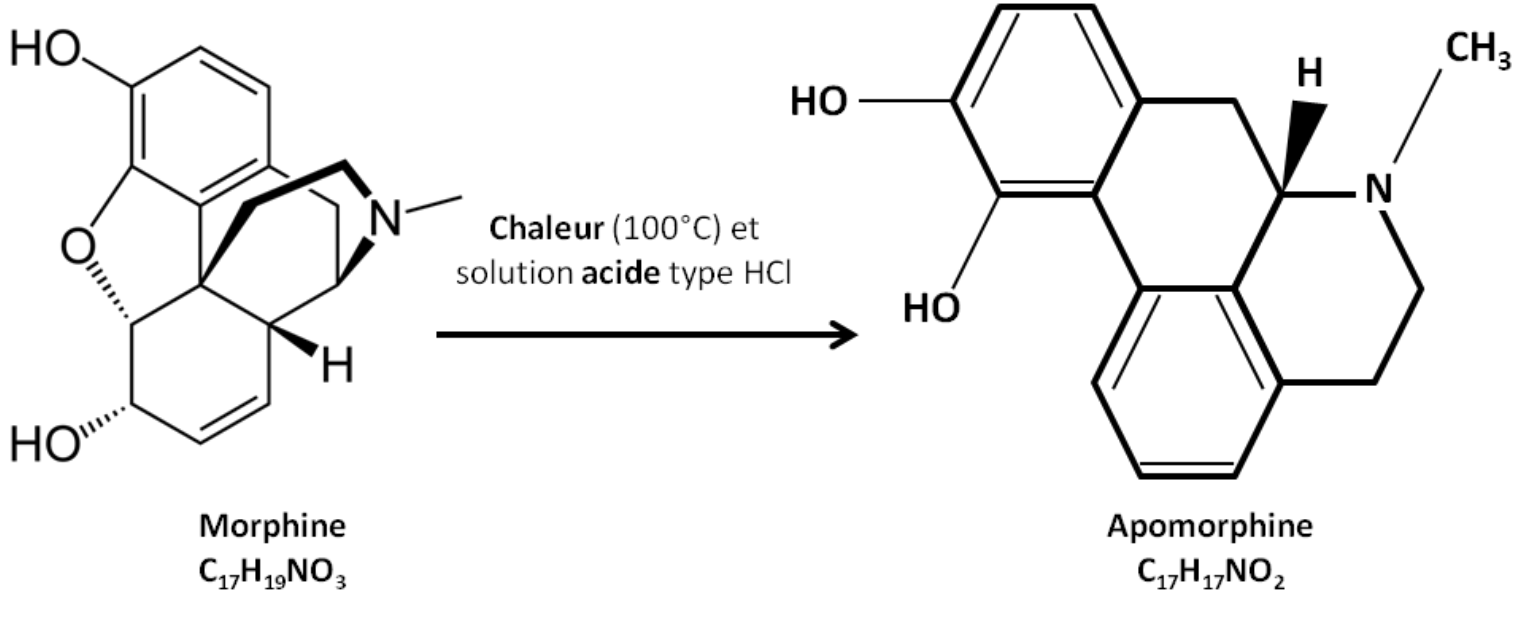

\section{Figure 1 : Synthèse d'apomorphine à partir de la molécule de morphine}

Du fait de sa structure, en particulier de la présence d'un motif catechol et donc de groupes hydroxyles $(-\mathrm{OH})$, la molécule est très sensible à l'oxydation : exposée à 
l'air, une solution d'apomorphine change de couleur en moins de $30 \mathrm{~min}$, ce qui signe la formation de quinones et une oxydation spontanée, catalysée par un pH élevé et la présence d'oxygène (Garrido et al, 2002 ; Anand Subramony, 2006 ; Haq et al, 2007). La solution se détériore rapidement au contact de l'air et prend une coloration verte, qui tâche les tissus en cas de contact (possibilité d'utiliser du jus de citron pour enlever les taches, Bowron, 2004). Lors de la préparation du médicament, il est donc nécessaire d'ajouter des antioxydants et des conservateurs, de type sodium ededate (plus connu sous le nom d'EDTA) et sulfites (métabisulfite de sodium). Ces substances sont susceptibles de provoquer des réactions allergiques voire un bronchospasme (Van Laar et al, 1998; Manson et al, 2001 ; Deleu et al, 2004 ; Résumé des Caractéristiques Produits (RCP) d'Apokinon ${ }^{\circledR} 10 \mathrm{mg} / \mathrm{mL}$, Laboratoire Aguettant, 2011 ; Ribaric, 2012). Une allergie aux sulfites est ainsi une contreindication à l'utilisation de l'apomorphine (Haq et al, 2007).

\section{Devenir de l'apomorphine dans l'organisme et considérations cliniques associées}

La pharmacocinétique de l'apomorphine suit un modèle à 2 compartiments (LeWitt, 2004) ; l'équilibre entre le compartiment sanguin et les compartiments cellulaires est atteint rapidement.

Sa faible biodisponibilité orale, qui se révèle inférieure à $4 \%$ et n'induit qu' 1 à $2 \%$ de l'activité obtenue par voie intraveineuse ou sous-cutanée (Gancher et al, 1991; Argiolas et Hedlund, 2001 ; Haq et al, 2007), a conduit à orienter la galénique de manière à éviter la circulation porte et l'estomac (Gancher et al, 1991 ; Koller et Stacy, 2004), du fait d'un fort effet de premier passage hépatique et d'une mauvaise absorption digestive. Si de nombreuses voies d'accès ont été étudiées, comme les voies parentérale, intranasale, sublinguale, rectale, transdermale (Lees, 1993 ; Colosimo et al, 1994 ; Poewe et Wenning, 2000 ; Argiolas et Hedlund, 2001 ; Manson et al, 2001 ; Koller et Stacy, 2004 ; Haq et al, 2007 ; Menon et Stacy, 2007 ; Stacy et Silver, 2008 ; Blandini et Armentero, 2014), la voie sous-cutanée (SC) dite parentérale extravasculaire, est considérée à l'heure actuelle comme la voie de référence (Hughes et al, 1993 ; Nicolle et al, 1993 ; Poewe et Wenning, 2000 ; Anand Subramony, 2006 ; Henriksen, 2014). L'injection, continue (pompe) ou discontinue ("stylo » injecteur), s'effectue dans l'hypoderme, préférentiellement au niveau de l'abdomen et de la partie supéro-externe des membres.

\section{Absorption et distribution}

Lors d'une administration SC, l'apomorphine agit très rapidement, en 5 à 15 minutes, avec une moyenne de 11 minutes (Stacy et Silver, 2008 ; Ribaric, 2012 ; Henriksen, 2014 ; Boyle et Ondo, 2015). Avec la forme stylo, utilisée pour lever les périodes off, une amélioration motrice est ressentie en moyenne 10 à 20 minutes après l'injection (Stacy et Silver, 2008). L'effet perdure pendant 40 à 90 minutes, avec un maximum de 120 minutes (Stacy, 2004 ; Factor, 2004 ; Haq et al, 2007 ; Stacy et Silver, 2008 ; Ribaric, 2012 ; Henriksen, 2014 ; Boyle et Ondo, 2015 ; Trenkwalder et al, 2015).

La demi-vie est de $33 \mathrm{~min}$, variant entre 30 et 90 min (Chen et al, 2005 ; Stacy et Silver, 2008 ; Ribaric, 2012 ; Chaudhuri et al, 2013). 
On retrouve une grande variabilité interindividuelle dans les paramètres pharmacocinétiques, notamment en termes de concentration plasmatique maximum (Cmax, dépendante de la dose) qui peut varier d'un facteur dix, d'aire sous la courbe (AUC) variant d'un facteur cinq selon les études, et du temps nécessaire pour atteindre la Cmax (Tmax, indépendant de la dose). Cette variabilité se traduit par des différences de doses efficaces selon les patients (LeWitt, 2004 ; Haq et al, 2007).

De nombreux paramètres sont à même d'expliquer cette variabilité : la dose, le volume et le mode d'administration de l'apomorphine, la région cutanée (absorption plus rapide au niveau de l'abdomen que des cuisses, du fait de la riche vascularisation sous-cutanée, Nicolle et al, 1993 ; Van Laar et al, 1998), la profondeur d'injection, la température cutanée (augmentation de la diffusion avec la chaleur), la vascularisation (flux sanguin), la quantité de graisse sous-cutanée ou encore les profils enzymatiques individuels, impliqués dans le métabolisme (Van der Geest et al, 1997 ; Deleu et al, 2004 ; Chen et al, 2005 ; Ribaric, 2012 ; Henriksen, 2014 ; Boyle et Ondo, 2015). Tous ces paramètres expliquent donc la variabilité des doses et de la réponse clinique entre patients (Steiger et al, 1992 ; Deleu et al, 2004 ; LeWiit, 2004 ; Drapier et Vérin, 2006). Cependant, une fois la dose individuelle optimale obtenue, on n'observe que très peu de variations au cours du traitement ; il existe donc une grande variabilité interindividuelle et une moindre variabilité intraindividuelle (Hughes et al 1993 ; Pietz et al 1998 ; LeWitt, 2004 ; Menon et Stacy, 2007 ; Ribaric, 2012 ; Wenzel et al, 2014).

Il est à noter par ailleurs que, selon l'origine de l'apomorphine et son mode de synthèse, les différentes formes présentes sur le marché peuvent varier en termes de puissance, de stabilité et/ou de pH (LeWitt, 2004 ; Haq et al, 2007), ce qui peut expliquer les différences de paramètres pharmacocinétiques et pharmacodynamiques observées dans les différentes études.

Des pics plasmatiques de 10 à $30 \mu \mathrm{g} / \mathrm{L}$ sont retrouvés dès 5 à 15 min après une administration SC et il faut 15 à 30 min pour atteindre les pics de concentration dans le liquide cérébro-spinal (LCS) (Chen et al, 2005 ; Haq et al, 2007 ; Wenzel et al, 2014).

De par sa structure polycyclique, l'apomorphine présente une lipophilie élevée qui lui permet de franchir la barrière hémato-encéphalique (BHE) et de conduire à des concentrations cérébrales six à huit fois supérieures à celles du plasma (Colosimo et al, 1994 ; Argiolas et Hedlund, 2001 ; Ribaric, 2012). Ces concentrations sont d'autant plus élevées et atteintes rapidement que l'apomorphine diffuse librement, contrairement à la lévodopa qui nécessite des transporteurs (Menon et Stacy, 2007 ; Ribaric, 2012). Par ailleurs, l'apomorphine ne nécessite pas la présence de terminaisons nerveuses présynaptiques fonctionnelles pour exercer son effet antiparkinsonien, à la différence de la lévodopa qui doit être transformée en dopamine au niveau de ces terminaisons (Ribaric, 2012).

L'effet antiparkinsonien est corrélé à la concentration extracellulaire striatale en apomorphine (LeWitt, 2004 ; Stocchi et Olanow, 2004 ; Haq et al, 2007 ; Chaudhuri et al, 2013). Cliniquement, l'apparition de bâillements (chez l'homme comme chez l'animal) signe l'atteinte cérébrale de l'apomorphine et le début d'une phase « on »; d'origine centrale, ces bâillements ne seraient pas liés à une sédation mais plutôt à un effet sur les récepteurs dopaminergiques D2 et/ou D3 (Bowron, 2004 ; Factor, 2004 ; Drapier et Vérin, 2006 ; Haq et al, 2007).

Bien qu'il ait été observé une augmentation du Tmax et une réduction du Cmax avec l'âge en administration sublinguale (SL), il n'y aurait pas nécessité d'ajuster la dose 
en fonction de l'âge des patients (Argiolas et Hedlund, 2001). Chez l'insuffisant rénal et/ou hépatique, on observe une augmentation de l'AUC et un allongement de la demi-vie d'élimination (RCP Apokinon® $10 \mathrm{mg} / \mathrm{mL}$, Laboratoire Aguettant, 2011). Par mesure de précaution, chez les patients présentant une défaillance organique, toute augmentation de dose devra donc être effectuée avec prudence (Chen et al, 2005).

L'apomorphine présente une très forte liaison aux protéines plasmatiques (principalement à l'albumine) de l'ordre de 90\% (Argiolas et Hedlund, 2001 ; LeWitt, 2004). II peut donc s'ensuivre une compétition avec d'autres médicaments (en particulier les acides liposolubles) pour ces sites de fixation, ce qui est susceptible de conduire à des interactions médicamenteuses (augmentation ou réduction de la forme libre, prolongation ou réduction de la durée de vie du médicament dans l'organisme). Cependant, aucune interaction de ce type n'a pour l'heure été recensée en médecine humaine.

\section{Métabolisme et élimination}

Le métabolisme de l'apomorphine est pluriel et associe une oxydation systémique (enzymatique et non enzymatique) et un catabolisme hépatique très largement prépondérants sur un faible métabolisme rénal (LeWitt, 2004 ; Menon et Stacy, 2007).

L'oxydation spontanée et $\mathrm{pH}$-dépendante de la structure catechol conduit à la formation de quinones électrophiles, qui interagissent avec les protéines et les composants tissulaires, ce qui impacte la pharmacocinétique de la molécule (Garrido et al, 2002).

Au niveau hépatique, l'apomorphine est principalement métabolisée par sulfatation, glucuronidation et glycuroconjugaison. La N-déméthylation conduit à la formation de norapomorphine qui est ensuite transformée en dérivés glucuronidés et sulfatés (Van der Geest et al, 1997 ; Argiolas et Hedlund, 2001 ; Ribaric, 2012). L'apomorphine est donc peu métabolisée par les isoformes du cytochrome P450 (enzymes ubiquitaires intervenant dans le métabolisme de substrats endogènes et exogènes), ce qui expliquerait le faible nombre d'interactions médicamenteuses, notamment avec les nombreux CYP-inhibiteurs et inducteurs (Henriksen, 2014). Si in vitro, l'apomorphine inhibe les CYP1A2, 2D6 et 3A4, in vivo, les concentrations plasmatiques seraient trop faibles (en tout cas pour la voie SL) pour avoir un impact chez l'homme (Argiolas et Hedlund, 2001).

La clairance de l'apomorphine est rapide (1 à $12 \mathrm{~L} / \mathrm{kg} / \mathrm{h}$, avec une demi-vie d'élimination de $40 \mathrm{~min}$ ), ce qui suggère une clairance extra-hépatique. Environ $0.3 \%$ d'une dose est excrétée sous forme inchangée.

Ni l'âge, le sexe, le poids, la sévérité et la durée d'évolution de la MP, la dose et la durée de traitement par lévodopa, ni même la durée de traitement par l'apomorphine ne semblent influencer ce paramètre (Chen et al, 2005, Haq et al, 2007 ; Menon et Stacy, 2007). Bien que la demi-vie d'élimination soit faible, on retrouve des traces de forme inchangée et de métabolites jusqu'à $12 \mathrm{~h}$ après l'arrêt de la perfusion chez un patient parkinsonien et jusqu'à $16 \%$ de la dose administrée sous forme inchangée ou métabolites dans les urines des patients (Van der Geest et al, 1997).

II a été noté des élévations doses dépendantes de l'urémie et de la créatinine sérique lors de l'administration orale de doses élevées (jusqu'à $1500 \mathrm{mg} / \mathrm{jour}$ ) et une azotémie dose-dépendante (vraisemblablement liée à l'accumulation de métabolites néphrotoxiques) se développant à long terme (Gancher et al, 1991 ; Tolosa et al, 
1998 ; Henriksen, 2014). Ces anomalies sont réversibles (Lees, 1993 ; Bowron, 2004). Une attention particulière pourra donc être apportée chez les patients en insuffisance rénale (Chen et al, 2005).

\section{Précautions d'emploi, interactions médicamenteuses et contre- indications}

L'apomorphine présente une sécurité d'emploi très satisfaisante, avec une absence de génotoxicité et de cancérotoxicité (Argiolas et Hedlund, 2001). Les données cliniques de suivi, sur des périodes de 1 à 108 mois indiquent qu'il n'y a pas de phénomène d'habituation, de tachyphylaxie ou de perte d'efficacité à long terme (Hughes et al, 1993 ; Pietz et al, 1998 ; Poewe et Wenning, 2000 ; Manson et al, 2001 ; Deleu et al, 2004 ; Pfeiffer et al, 2007 ; Haq et al, 2007 ; Stacy et Silver, 2008 ; Hattori et al, 2014 ; Henriksen, 2014 ; Wenzel et al, 2014). De même, l'apomorphine est bien tolérée sur de longues périodes (Pietz et al, 1998 ; Tyne et al, 2004), sans baisse d'efficacité même en cas de traitement antérieur de longue durée par lévodopa (Factor, 2004).

Son efficacité en dose aiguë semble cependant diminuer avec la sévérité de la maladie et l'intensité des lésions (dégénérescence neuronale nigrostriatale et altérations consécutives post-synaptiques) : moindre durée d'efficacité (28 min contre $52 \mathrm{~min}$ ), réduction de la fenêtre thérapeutique et apparition de dyskinésies à des doses plus faibles (Metman et al, 1997 ; Haq et al, 2007 ; Menon et Stacy, 2007 ; Rambour et al, 2014).

Peu d'interactions pharmacologiques à incidence clinique majeure ont été rapportées à ce jour (Deleu et al, 2004). Dans le cadre de la MP, l'apomorphine peut être associée à la lévodopa et à l'entacapone sans modification des paramètres pharmacodynamiques ou pharmacocinétiques: elle présente donc une grande sûreté d'utilisation en administration aiguë (Deleu et al, 2004 ; Zijlmans et al, 2004). II est à noter par ailleurs qu'une thérapeutique concomitante par antiagrégant ou anticoagulant ne constitue pas une contre-indication (Drapier et Vérin, 2006).

Quelques interactions médicamenteuses sont cependant à prendre en compte. On recense notamment des interactions avec certains antihypertenseurs et les dérivés nitrés (Argiolas et Hedlund, 2001; Montorsi, 2003), ou encore l'alcool, du fait de l'effet combiné sur la pression artérielle (hypotension).

L'utilisation concomitante d'antagonistes 5-HT3 (ex : granisetron, ondansetron) est à proscrire, du fait du risque d'hypotension sévère, voire de perte de conscience et de syncope (Chen et al, 2005 ; Menon et Stacy, 2007).

Par ailleurs, à une dose supérieure à $6 \mathrm{mg}$, l'apomorphine est susceptible d'allonger l'espace QT : il faut rester attentif aux prescriptions parallèles de médicaments entraînant le même effet cardiaque, comme certains psychotropes, antagonistes 5HT3 ou encore l'amiodarone, voire même la dompéridone (Chen et al, 2005 ; RCP Apokinon $\AA 10 \mathrm{mg} / \mathrm{mL}$, Laboratoire Aguettant, 2011). On déconseillera également son utilisation chez les patients prédisposés aux torsades de pointes par la présence d'une hypokaliémie, d'une bradycardie ou d'une hypomagnésémie (Chen et al, 2005). Une vigilance particulière est de mise chez les patients susceptibles de présenter des troubles du rythme du fait de leur âge ou de toute autre condition (Kaminioti et al, 2013). Un examen ECG est ainsi nécessaire avant l'introduction de la pompe à apomorphine (Trenkwalder et al, 2015). 
Les contre-indications à l'administration d'apomorphine sont une insuffisance hépatocellulaire sévère, une hypersensibilité à l'apomorphine ou à l'un des composants, comme le métabisulfite de sodium (Chen et al, 2005 ; Menon et Stacy, 2007).

Les effets de l'apomorphine pendant la grossesse n'ayant pas été étudiés, on déconseillera son usage du fait de l'absence de données sur un éventuel effet malformatif ou foetotoxique (Chen et al, 2005; RCP Apokinon® $10 \mathrm{mg} / \mathrm{mL}$, Laboratoire Aguettant, 2011). L'apomorphine étant excrétée dans le lait maternel, on déconseillera son utilisation pendant l'allaitement (RCP Apokinon® $10 \mathrm{mg} / \mathrm{mL}$, Laboratoire Aguettant, 2011). Cependant, l'âge de la population concernée rend la survenue d'une grossesse peu probable.

\section{Ce qu'il faut retenir de l'administration SC :}

- $\quad$ Apparition d'un effet en 11 min, pour une durée d'environ 60 min

- $\quad$ Grande variabilité interindividuelle mais peu de variabilité intraindividuelle

- Lipophilie élevée, diffusion libre à travers la BHE, effet corrélé aux concentrations cérébrales

- $\quad$ Pas d'implication des CYP450 dans le métabolisme donc pas d'interactions médicamenteuses à type d'induction/inhibition avec les médicaments à marge thérapeutique étroite

- $\quad$ Sensibilité élevée à l'oxydation

Prudence chez l'insuffisant hépatique, rénal et cardiaque

Contre-indication: hypersensibilité (apomorphine, sulfites), insuffisance hépatocellulaire sévère, grossesse/allaitement

Interactions : alcool, antagonistes 5-HT3 (setrons), dérivés nitrés, hypotenseurs, médicaments allongeant l'espace QT

Pas de perte d'effet à long terme, bonne tolérance

\section{Apomorphine et récepteurs : intérêt clinique}

De structure très proche de celle de la dopamine (Ernst, 1965), l'apomorphine comprend un noyau catechol, qui permet la liaison aux récepteurs dopaminergiques, et une structure 2-amino-tetralein qui déterminerait sa propriété d'agoniste dopaminergique (Menon et Stacy, 2007 ; Boyle et Ondo, 2015). Plus précisément, l'apomorphine comprend le pharmacophore de la dopamine, soit deux groupements hydroxyles séparés du noyau azoté par une distance de 0,7 à 0,8nm (Figure 2). Ces caractéristiques en font un agoniste dopaminergique non sélectif, c'est-à-dire reconnaissant l'ensemble des récepteurs dopaminergiques (cinq sous-types), qu'ils soient pré ou post-synaptiques. 

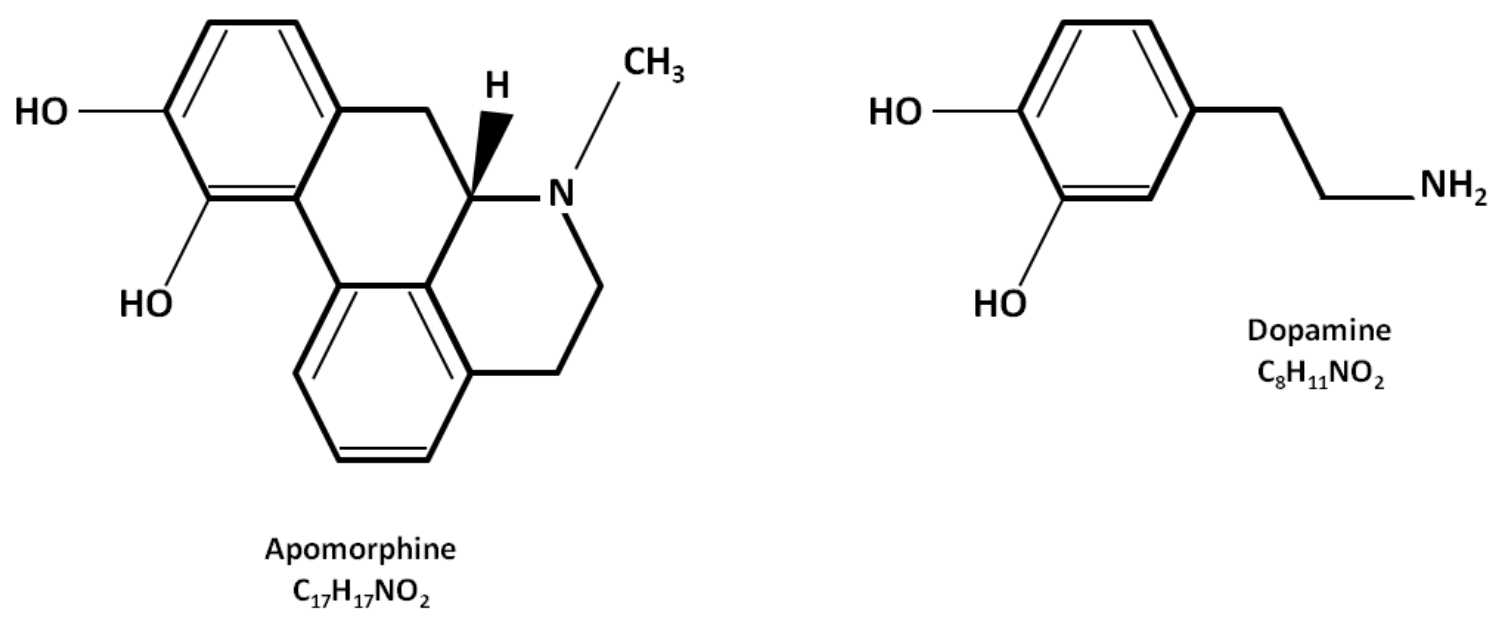

\section{Figure 2 : Analogies structurales entre l'apomorphine et la dopamine}

\section{Action dopaminergique}

L'apomorphine est une molécule chirale (asymétrique); les récepteurs dopaminergiques étant stéréo-sélectifs, les énantiomères $R$ et $S$ présentent des activités et des affinités différentes malgré des propriétés physicochimiques identiques (Sam et al, 1997). L'énantiomère $R$ agit ainsi comme un agoniste dopaminergique (Gassen et al, 1996 ; Anand Subramony, 2006 ; Hara et al, 2006) et induit principalement des effets moteurs et des stéréotypies chez le rongeur. C'est la seule forme active cliniquement (Deleu et al, 2004; LeWitt, 2004; Anand Subramony, 2006), l'énantiomère $S$ étant dépourvu d'activité agoniste et agissant plutôt comme un antagoniste dopaminergique (Van der Geest et al, 1997 ; LeWitt, 2004).

Les récepteurs dopaminergiques appartiennent à la superfamille des récepteurs couplés aux protéines $\mathrm{G}$. Ils présentent une répartition régionale, une biochimie et des propriétés pharmacologiques différentes qui les rendent uniques (Deleu et al, 2004). Ils sont classés en deux groupes, selon la protéine $G$ qui leur est associée (Gs, Gi et Go) et la présence ou non d'introns dans la séquence codante de gènes (Sokoloff et al, 1993). Les récepteurs dits $D 1$-like, sont ainsi couplés positivement à l'enzyme adénylate cyclase (AC) par une protéine Gs, couplage qui entraîne l'activation des structures porteuses de ces récepteurs. A l'inverse, les récepteurs D2-like sont couplés à l'inhibition de l'AC par des protéines Gi ou Go. Les récepteurs D1 et D2 sont les plus répandus au niveau cérébral (Sokoloff et al, 1993).

Les récepteurs $\mathrm{D} 1$ et $\mathrm{D} 5$, dits $D 1$-like (codés par des gènes des chromosomes 5 pour le récepteur D1 et 4 pour le D5, Seeman et Van Tol, 1994), sont donc couplés positivement à l'AC et activés par la dopamine. Ils sont localisés sur les membranes postsynaptiques des neurones, majoritairement au niveau du néostriatum, du noyau accumbens, du tubercule olfactif, de l'amygdale, de l'hypothalamus et de l'hippocampe. Ils appartiendraient à la voie directe, excitatrice, promouvant les mouvements adaptés au comportement, avec une projection neuronale vers le globus pallidus interne (GPi) et la substance noire (SN) pars reticulata (Nyholm, 2007).

Les récepteurs D2, D3 et D4, dits D2-like, sont quant à eux majoritairement localisés dans les régions limbiques et striatales, au niveau présynaptique (autorécepteurs) et 
postsynaptique (Deleu et al, 2004). L'activation membranaire de ces récepteurs inhibe la production d'AMPc par l'AC et conduit à l'inhibition des neurones qui les supportent. Du fait de la présence d'introns dans les séquences codantes des gènes, les récepteurs D2-like présentent tous plusieurs variants chez l'homme (Sokoloff et al, 1993 ; Seeman et Van Tol, 1994 ; Ribaric, 2012). Ils appartiendraient à la voie indirecte, inhibitrice, qui vise à stopper les mouvements inappropriés, avec une projection neuronale vers le globus pallidus externe (GPe) et le noyau subthalamique ou NST (Nyholm, 2007).

En conditions expérimentales et cliniques, ce sont les récepteurs D2-like qui permettent un effet antiparkinsonien, par activation de la voie directe et inhibition de la voie indirecte striatopallidale (Deleu et al, 2004 ; Menon et Stacy, 2007).

Ubiquitaire, l'apomorphine stimule donc les récepteurs pré et post-synaptiques $D 1$ like (D1 et D5) et D2-like (D2, D3 et D4), alors que la plupart des agonistes dopaminergiques stimulent essentiellement les récepteurs de type D2 (Colosimo et al, 1994 ; Muguet et al, 1995 ; Pietz et al, 1998 ; LeWitt, 2004 ; Chen et al, 2005 ; Ribaric, 2012). Une telle stimulation des cinq sous-types de récepteurs dopaminergiques produit donc par synergie un effet antiparkinsonien plus puissant que la stimulation isolée de chaque classe de récepteurs, la stimulation concomitante des récepteurs D1 augmentant les effets de celle des D2 (Ellis et al, 1997 ; Stacy, 2004 ; Deleu et al, 2004 ; Chen et al, 2005).

Les conclusions de multiples études chez le rongeur et de quelques études cliniques laissent supposer un effet biphasique de l'apomorphine, avec des effets parfois opposés selon les doses utilisées (Ellis et al, 1997 ; Ruzicka et al, 1994 ; Ribaric, 2012). Ils sont attribués à l'effet sélectif de la molécule sur les récepteurs dopaminergiques présynaptiques (autorécepteurs) et postsynaptiques, avec une stimulation préférentielle et inhibitrice des autorécepteurs lors de l'administration de faibles doses, vraisemblablement consécutive à une différence de sensibilité (Ruzicka et al, 1994). II semblerait ainsi que l'apomorphine hydrochloride (forme sel) sous-cutanée à la dose de 3.5-5.0 $\mu \mathrm{g} / \mathrm{kg}$ soit une dose présynaptique, alors que 7.0 $\mu \mathrm{g} / \mathrm{kg}$ soit une dose postsynaptique (Dépatie et Lal, 2001). Par ailleurs, il a été suggéré une plus grande sensibilité des récepteurs mésocorticaux par rapport aux récepteurs nigrostriataux (Ruzicka et al, 1994).

II est à noter que l'apomorphine présente une affinité différentielle pour les différents récepteurs dopaminergiques : elle possède la plus grande affinité pour les récepteurs D4, suivis des récepteurs D3 et D5 (affinité équivalente pour ces deux récepteurs) puis les D2 et enfin les D1 (Argiolas et Hedlund, 2001 ; LeWitt, 2004 ; Haq et al, 2007 ; Poewe, 2009 ; Sujith et Lane 2009). Son profil de stimulation des récepteurs dopaminergiques est similaire à celui de la dopamine et proche du gold-standard qu'est la lévodopa (Kempster et al, 1990 ; Stacy, 2004 ; Poewe, 2009 ; Hattori et al, 2014 ; Boyle et Ondo, 2015). Comparée à la dopamine, l'apomorphine présente une affinité similaire pour les D1, une affinité bien plus marquée pour les D2 (100 fois supérieure, selon Tellez et al, 2006) et les D4, et une affinité 7 fois plus faible pour les D3 (Argiolas et Hedlund, 2001 ; Dépatie et Lal, 2001).

\section{Action non dopaminergique}

L'apomorphine n'est pas qu'un simple agoniste dopaminergique; elle active également des récepteurs sérotoninergiques et adrénergiques (Sam et al, 1997 ; LeWitt, 2004 ; Sujith et Lane, 2009 ; Ribaric et al, 2012 ; Wenzel et al, 2014). 
Elle interagirait ainsi avec les récepteurs 5HT1A/2A/2B/2C (LeWitt, 2004 ; Menon et Stacy, 2007 ; Ribaric, 2012 ; Wenzel et al, 2014). En culture cellulaire (cellules de neuroblastomes murins N1E-115), il a été retrouvé une interaction avec le récepteur 5-HT3, connu pour être impliqué dans la régulation de l'activité locomotrice et dans la libération de dopamine (Van Hooft et Vijverberg, 1998). L'énantiomère $R$ a ainsi un effet agoniste 5-HT3, c'est à dire qu'il augmente la libération de dopamine (bien qu'une exposition prolongée conduise à une inhibition des récepteurs), alors que l'énantiomère $S$ est quant à lui un antagoniste non compétitif (Van Hooft et Vijverberg, 1998). Cette interaction est d'autant plus intéressante que, dans le cerveau normal, les systèmes dopaminergiques et sérotoninergiques interagissent au niveau de la substance noire et du noyau du raphé (Chaudhuri et al, 2013).

En ce qui concerne les adrénorécepteurs, les affinités sont plus controversées selon les auteurs : $\alpha 1 \mathrm{~B} / 1 \mathrm{D} / 2 \mathrm{~A} / 2 \mathrm{~B} / 2 \mathrm{C}$ (Ribaric, 2012), uniquement $\alpha 2 \mathrm{~A} / \mathrm{B} / \mathrm{C}$ (Blandini et Armentero, 2014) ou a1D/2B/2C (LeWitt, 2004 ; Haq et al, 2007 ; Menon et Stacy, 2007 ; Wenzel et al, 2014).

\section{Intérêt dans la maladie de Parkinson}

La MP est une pathologie multidimensionnelle chronique, évolutive et complexe, induisant des altérations centrales et périphériques des systèmes dopaminergiques, noradrénergiques, sérotoninergiques et cholinergiques, mais aussi glutamatergiques et GABAergiques (Brotchie, 2005 ; Nyholm, 2007 ; Blandini et Armentero, 2014). On voit donc l'intérêt d'utiliser l'apomorphine dans cette indication, de par son profil pharmacologique particulier et ses interactions avec diverses familles de récepteurs (LeWitt, 2004).

Autre point d'intérêt dans la MP, l'apomorphine aurait également un effet inhibiteur des monoamines oxydases (MAO-A et MAO-B), enzymes impliquées dans le catabolisme des monoamines telles que la dopamine et la sérotonine (Deleu et al, 2004).

Un effet neuroprotecteur multifactoriel (Deleu et al, 2004 ; Hara et al, 2006 ; Vaglini et al, 2008 ; Wenzel et al, 2014) a été démontré in vitro et in vivo lors d'études précliniques (Blandini et Armentero, 2014). II pourrait être médié par les récepteurs D2 présynaptiques (Deleu et al, 2004) mais également par des voies non dopaminergiques (Ribaric, 2012). En culture cellulaire, l'apomorphine présente un effet dose-dépendant : à faible dose, elle est protectrice (effet neurotrophique) mais à forte dose, elle induit une cytotoxicité à laquelle les neurones dopaminergiques, connus pour être plus fragiles, sont particulièrement sensibles (Vaglini et al, 2008). Cette toxicité semble liée à l'auto-oxydation de l'apomorphine en dérivés de type quinones, qui induiraient une apoptose. Cependant, les doses nécessaires pour reproduire cet effet in vivo sont bien trop élevées pour que cela soit observé en conditions thérapeutiques (Vaglini et al, 2008).

L'apomorphine aurait également un effet protecteur contre le stress oxydant (SO) et serait antiradicalaire (Gassen et al, 1996 ; Hara et al, 2006 ; Himeno et al, 2011 ; Ribaric, 2012 ; Blandini et Armentero, 2014, Boyle et Ondo, 2015). Elle agit comme un capteur de radicaux libres, ce qui peut présenter un intérêt majeur à l'heure où l'on s'interroge sur l'hypothèse inflammatoire de la MP (Collins et al, 2012). Elle activerait ainsi la voie Nrf2/ARE, un facteur de transcription de la réponse au stress oxydant (Hara et al, 2006). II a par ailleurs été retrouvé un effet antiprolifératif sur quelques lignées de cellules tumorales (Vaglini et al, 2008). Ces résultats 
expérimentaux sont tangibles mais aucun " disease-modifying effect " n'a pour l'heure été retrouvé en clinique (Deleu et al, 2004 ; Ribaric, 2012 ; Blandini et Armentero, 2014). Suite à ces observations, des pistes quant à une application éventuelle dans la maladie d'Alzheimer ont récemment été proposées (Himeno et al, 2011 ; Ribaric et al 2012).

\section{Ce qu'il faut retenir à propos des récepteurs :}

Structure similaire à la dopamine, chirale (énantiomère $\mathrm{R}$, actif) Agoniste dopaminergique le plus puissant (synergie) et polymorphe Activité pré et post synaptique sur tous les récepteurs dopaminergiques, avec une affinité différentielle : D4>D3/D5>D2>D1

Interaction avec les systèmes sérotoninergiques (récepteurs $5 \mathrm{HT} 1 \mathrm{~A} / 2 \mathrm{~A} / 2 \mathrm{~B} / 2 \mathrm{C}$ ) et adrénergiques (récepteurs a1B/1D/2A/2B/2C) Effet neuroprotecteur potentiel

\section{Neurophysiologie et Neuroimagerie}

Les études les plus nombreuses publiées à ce jour dans le champ de l'imagerie cérébrale et de l'apomorphine concernent principalement le rat, le métabolisme glucidique et datent des années 1980. Ces travaux montrent que l'administration d'agonistes dopaminergiques induit des modifications métaboliques dans les voies directes et indirectes (Conti et al, 2001).

Peu de travaux se sont intéressés aux effets cérébraux de l'apomorphine chez l'homme et les rares données sont contradictoires, allant d'une absence d'effet à l'induction de modifications corticales et sous-corticales (Broussolle et al, 1993 ; Montorsi et al, 2003 ; Ribaric, 2012). L'un des effets rapportés de l'apomorphine consisterait cependant à atténuer l'inhibition du thalamus par le $\mathrm{GPi}$, avec une activation corticale subséquente (Deleu et al, 2004 ; Ribaric, 2012). Des enregistrements électrophysiologiques au décours de chirurgies cérébrales (pallidotomie, stimulation cérébrale profonde) suggèrent également que l'apomorphine diminuerait l'activité du GPi et du STN et augmenterait l'activité du GPe (Lozano et al, 2000 ; Stacy, 2004).

\section{Conclusion et perspectives}

En administration sous-cutanée, l'apomorphine agit rapidement et se distribue dans tout le corps avec une prévalence au niveau cérébral. L'étude de la pharmacocinétique permet d'expliquer la grande variabilité interindividuelle en termes de posologie efficace et doit encourager le clinicien à ajuster la dose et la durée de stimulation selon la réactivité de son patient, dans une approche personnalisée.

La répartition des récepteurs dopaminergiques au niveau des aires cérébrales et l'intrication entre les systèmes dopaminergiques et sérotoninergiques permettent d'envisager l'éventualité d'effets moteurs et comportementaux multiples consécutifs à l'administration d'apomorphine. 
Agoniste dopaminergique polymorphe, agoniste sérotoninergique et adrénergique, molécule neuroprotectrice, l'apomorphine se révèle donc d'un grand intérêt au sein de la pharmacopée antiparkinsonienne.

\section{Références bibliographiques}

Anand Subramony J. Apomorphine in dopaminergic therapy. Review. Molecular Pharmaceutics 2006 Vol 3, No4, 380-385.

Antonini A. Apomorphine and Levodopa infusion therapies for advanced Parkinson's disease. Journal of Movement Disorders 2009;2:4-9.

Argiolas $A$, Hedlund $H$. The pharmacology and clinical pharmacokinetics of apomorphine SL. BJU International (2001), 88(Suppl 3), 18-21.

Blandini F, Armentero MT. Dopamine receptor agonists for Parkinson's disease. Expert Opin. Investig. Drugs (2014) 23(3):387-410.

Bowron A. Practical considerations in the use of apomorphine injectable. Neurology 2004;62(Suppl 4):S32-S36.

Boyle A, Ondo W. Role of apomorphine in the treatment of Parkinson's Disease. CNS Drugs (2015) 29:83-89.

Brotchie JM. Nondopaminergic mechanisms in levodopa-induced dyskinesia. Movement disorders, Vol.20, No.8, 2005, pp.919-931. Research Review.

Broussolle E, Cinotti L, Pollak P, Landais P, Le Bars D, Galy G, Lavenne F, Khalfallah $Y$, Chazot $G$, Mauguière $F$. Relief of akynesia by apomorphine and cerebral metabolic changes in Parkinson's disease. Movement Disorders Vol 8, No.4, 1993, pp. 459-462.

Chaudhuri KR, Rizos A, Sethi KD. Motor and nonmotor complications in Parkinson's disease: an argument for continuous drug delivery? J Neural Transm (2013) 120:1305-1320.

Chen JJ, Obering C. A review of intermittent subcutaneous apomorphine injections for the rescue management of motor fluctuations associated with advanced Parkinson's disease. Clin Ther. 2005; 27:1710-1724.

Collins LM, Toulouse A, Connor TJ, Nolan YM. Contributions of central and systemic inflammation to the pathophysiology of Parkinson's disease. Neuropharmacology 62 (2012) 2154-2168.

Colosimo C, Merello M, Albanese A. Clinical usefulness of apomorphine in movement disorders. Clinical Neuropharmacology 1994 Vol.17, No.3, pp. 243-259. 
Conti G, Blandini F, Tassorello C, Giubilei F, Foranai F, Zocchia A, Orzia F. Intrastriatal injection of D1 or D2 dopamine agonists affects glucose utilization in both the direct and indirect pathways of the rat basal ganglia. Neuroscience Letters 309 (2001) 161-164.

Defebvre L, Vérin M. La maladie de Parkinson. 3ème édition (2015) Elsevier Masson, France. 240 pages. ISBN 9782294742323.

Deleu D, Hanssens Y, Northway MG. Subcutaneous apomorphine: an evidencebased review of its use in Parkinson's disease. Drugs Aging 2004;21(11):687-709.

Dépatie L, Lal S. Apomorphine and the dopamine hypothesis of schizophrenia: a dilemma? J Psychiatry Neurosci 2001;26(3):203-20.

Drapier S, Vérin M. L'apomorphine en perfusion sous-cutanée continue dans le traitement de la maladie de Parkinson. Rev Neurol (Paris) 2006 ; $162: 10,1019$ 1023.

Ellis C, Lemmens G, Parkes JD, Abbott RJ, Pye IF, Leigh PN, Chaudhuri KR. Use of apomorphine in Parkinsonian patients with neuropsychiatric complications to oral treatment. Parkinsonism \& Relatde disorders Vol.3, No.2, pp.103-107, 1997.

Factor SA. Intermittent subcutaneous apomorphine therapy in Parkinson's disease. Literature review. Neurology 2004;62(Suppl 4):S12-S17.

Gancher ST, Nutt JG, Woodward WR. Absorption of apomorphine by various routes in Parkinsonism. Movement Disorders Vol 6, No3, 1991, pp 212-216.

Garrido JPMJ, Delerue-matos C, Borges F, Macedo TRA, Oliveira-Brett AM. New insights into the oxidation pathways of apomorphine. J. Chem. Soc., Perkin Trans. 2, 2002, 1713-1717

Gassen M, Glinka Y, Pinchasi B, Youdim MBH. Apomorphine is a highly potent free radical scavenger in rat brain mitochondrial fraction. European Journal of Pharmacology 308 (1996) 219-225.

Haq IU, Lewitt PA, Fernandez HH. Apomorphine therapy in Parkinson's disease: a review. Expert Opin. Pharmacother. (2007) 8(16):2799-2809.

Hara H, Ohta M, Adachi T. Apomorphine protects against 6-hydroxydopamineinduced neuronal cell death through activation of the Nrf2-ARE pathway. Journal of Neuroscience Research 84:860-866 (2006).

Hattori N, Nomoto M, 6500-004 Study Group. Sustained efficacy of apomorphine in Japanese patients with advanced Parkinson's Disease. Parkinsonism and related disorders 20 (2014) 819-823.

Henriksen T. Clinical insights into use of apomorphine in Parkinson's disease: tools for clinicians. Neurodegen. Dis. Manage. (2014) 4(3), 271-282. 
Himeno E, Ohyagi Y, Ma L, Nakamura N, Miyoshi K, Sakae N, Motomura K, Soejima N, Yamasaki R, Hashimoto T, Tabira T, LaFerla FM, Kira Jl. Apomorphine treatment in Alzheimer mice promoting Amyloid- $\beta$ degradation. Ann Neurol $2011 ; 69$ :248-256.

Hughes AJ, Bishop S, Kleedorfer B, Turjanski N, Fernandez W, Lees AJ, Stern GM. Subcutaneous apomorphine in Parkinson's disease: response to chronic administration for up to five years. Movement Disorders Vol.8, No.2, 1993, pp.165170.

Kaminioti AN, Nikitas GT, Terlis AK, Manolis AG, Thomaides T, Panousopoulou AN. Ventricular bigeminy after Subcutaneous administration of apomorphine in a patient with refractory Parkinson's disease : a case report. Journal of Movement disorders 2013;6:9-12.

Kempster PA, Frankel JP, Stern GM, Lees AJ. Comparison of motor response to apomorphine and levodopa in Parkinson's disease. J Neurol Neurosurg Psychiatry. 1990;53:1004-1007.

Koller W, Stacy M. Other formulations and future considerations for apomorphine for subcutaneous injection therapy. Neurology 2004;62(Suppl 4):S22-S26.

Lees AJ. Dopamine agonists in Parkinson's disease: a look at apomorphine. Review Fundam Clin Pharmacol (1993) 7, 121-128

LeWitt PA. Subcutaneously administered apomorphine. Pharmacokinetics and metabolism. Neurology 2004; 62 (Suppl 4):S8-S11.

Lozano AM, Lang AE, Levy R, Hutchison W, Dostrovsky J. Neuronal recordings in Parkinson's disease patients with dyskinesias induced by apomorphine. Ann Neurol 2000; 47(Suppl.):141-146.

Manson AJ, Hanagasi H, Turner K, Patsalos PN, Carey P, Ratnaraj N, Lees AJ. Intravenous apomorphine therapy in Parkinson's disease. Clinical and pharmacokinetic observations. Brain (2001), 124, 331-340.

Menon R, Stacy M. Apomorphine in the treatment of Parkinson's disease. Expert Opin. Pharmacother. (2007) 8 (12):1941-1950.

Metman LV, Locatelli ER, Bravi D, Mouradian MM, Chase TN. Apomorphine responses in Parkinson's disease and the pathogenesis of motor complications. Neurology 1997; 48:369-372.

Montorsi F, Perani D, Anchisi D, Salonia A, Scifo P, Rigiroli P, Zanoni M, Heaton JPW, Rigatti P, Fazio F. Apomorphine-induced brain modulation during sexual stimulation: a new look at central phenomena related to erectile dysfunction. International Journal of Impotence Research 15 (2003) 203-209. 
Muguet D, Broussolle E, Chazot G. Apomorphine in patients with Parkinson's disease. Biomed Pharmacother 49(4) (1995) 197-209. Review.

Nicolle E, Pollak P, Serre-Debeauvais F, Richard P, Gervason CL, Broussolle E, Gavend M. Pharmacokinetics of apomorphine in parkinsonian patients. Fundam Clin Pharmacol (1993) 7, 245-252.

Nyholm D. The rationale for continuous dopaminergic stimulation in advanced Parkinson's disease. Parkinsonism and Related Disorders 13 (2007) S13-S17.

Pietz K, Hagell P, Odin P. Subcutaneous apomorphine in late stage Parkinson's disease: a long term follow up. J Neurol Neurosurg Psychiatry 65 (1998) 709-716.

Pfeiffer RF, Gutmann L, Hull Jr. KL, Bottini PB, Sherry JH, The APO302 study investigators. Continued efficacy and safety of subcutaneous apomorphine in patients with advanced Parkinson's disease. Parkinsonism and Related Disorders 13 (2007) 93-100.

Poewe W, Wenning GK. Apomorphine: an underutilized therapy for Parkinson's disease. Review Movement Disorders Vol.15, No.5, 2000, pp789-794.

Rambour M, Moreau C, Salleron J, Devos D, Kreisler A, Mutez E, Simonin C, Annic A, Dujardin K, Destée A, Defebvre. Le traitement par apomorphine en perfusion continue sous-cutanée dans la maladie de Parkinson: analyse rétrospective de 81 patients. Revue Neurologique 170 (2014) 205-215.

Ribaric S. The pharmacological properties and therapeutic use of apomorphine. Review. Molecules, 2012, 17, 5289-5309.

Ruzicka E, Roth J, Spackova N, Mecir P, Jech R. Apomorphine induced cognitive changes in Parkinson's disease. J Neurol Neurosurg Psychiatry, 1994; 57:998-1001.

Sam E, Sarre S, Michotte Y, Verbeke N. Distribution of apomorphine enantiomers in plasma, brain tissue and striatal extracellular fluid. European Journal of Pharmacology 329 (1997) 9-15.

Seeman P, Van Tol MH. Dopamine receptor pharmacology. Trends Pharmacol Sci 1994; 15:264-70.

Sokoloff $P$, Martres MP, Schwartz JC. La famille des récepteurs de la dopamine. Médecine/Sciences, 9, 12, 1993

Stacy M, Silver D. Apomorphine for the acute treatment of "off" episodes in Parkinson's disease. Review. Parkinsonism and related Disorders 14 (2008) 85-92

Stacy M. Apomorphine. North American Clinical Experience. Neurology 2004; 62(Suppl 4):S18-S21 
Steiger MJ, Quinn NP, Marsden CD. The clinical use of apomorphine in Parkinson's disease. J Neurol (1992) 239: 389-393.

Stocchi F, Olanow CW. Continuous dopaminergic stimulation in early and advanced Parkinson's disease. Neurology 2004;62(Suppl 1):S56-S63.

Sujith OK, Lane C. Therapeutic options for continuous dopaminergic stimulation in Parkinson's disease. Therapeutic Advances in Neurological disorders (2009) 2(2) 105-113.

Tolosa E, Marti MJ, Valldeoriola F, Molinuevo JL. History of levodopa and dopamine agonists in Parkinson's disease treatment. Neurology 1998;50(Suppl6):S2-S10.

Trenkwalder C, Chaudhuri KR, Garcia Ruiz PJ, LeWitt P, Katzenschlager R, SixelDöring F, Henriksen T, Sesar A, Poewe W, on behalf of an Expert Consensus Group for the Use of Apomorphine in Parkinson's Disease, Baker M, Ceballos-Baumann A, Deuschl G, Drapier S, Ebersbach G, Evans A, Fernandez H, Isaacson S, van Laar T, Lees A, Lewis S, Castrillo JCM, Martinez-Martin P, Odin P, O'Sullivan J, Tagaris G, Wenzel K. Expert consensus group report on the use of apomorphine in the treatment of Parkinson's disease-Clinical practice recommendations. Parkinsonism and related disorders 21 (2015) 1023-1030

Tyne HL, Parsons J, Sinnott A, Fox SH, Fletcher NA, Steiger MJ. A 10 year retrospective audit of long-term apomorphine use in Parkinson's disease. J Neurol (2004) 251:1370-1374.

Vaglini F, Pardini C, Viaggi C, Caramelli A, Corsini GU. Apomorphine offers new insight into dopaminergic neuron vulnerability in mesencephalic cultures. Neuropharmacology 55 (2008) 737-742.

Van der Geest, R, Kruger P, Gubbens-Stibbe JM, Van Laar T, Bodde HE, Danhof M. Assay of R-apomorphine, S-apomorphine, apocodeine, isoapocodeine and their glucuronide and sulfat conjugates in plasma and urine of patients with Parkinson's disease. Journal of Chromatography B, 702 (1997) 131-141.

Van Laar T, Van Hilten B, Neef C, Rutgers AWF, Pavel S, Brujin JA. The role of EDTA in provoking allergic reactions to subcutaneous infusion of apomorphine in patients with Parkinson's disease: a histologic study. Movement disorders, 13,1,1998, pp52-55

Van Hooft JA, vijverberg HPM. Agonist and antagonist effects of apomorphine enantiomers on 5-HT3 receptors. Neuropharmacology 37 (1998) 259-264

Walter E, Odin P. Cost-effectiveness of continuous subcutaneous apomorphine in the treatment of Parkinson's disease in the UK and Germany. J Med Econ 2015;18:15565. 
Wenzel K, Homann CK, Fabbrini G, Colosimo C. The role of subcutaneous infusion of apomorphine in Parkinson's disease. Expert Rev. Neurother. 14(7), 833-843 (2014).

Zagnoli F, Rouhart F. Maladie de Parkinson. 2ème édition (2006). Doin, Collection conduites, France. 209 pages. ISBN 2-7040-1212-1.

Zijlmans JCM, Debilly B, Rascol O, Lees AJ, and Durif F. Safety of entacapone and apomorphine coadministration in Levodopa-Treated Parkinson's disease Patients: pharmacokinetic and pharmacodynamic results of a multicenter, double-blind, placebo-controlled, cross-over study. Movement Disorders Vol.19, No.9, 2004, pp. 1006-1011. 